\title{
Análisis de la incidencia de la edad en la competencia digital del profesorado preuniversitario español
}

\section{Analysis of the incidence of age in the digital competence of Spanish pre-university teachers}

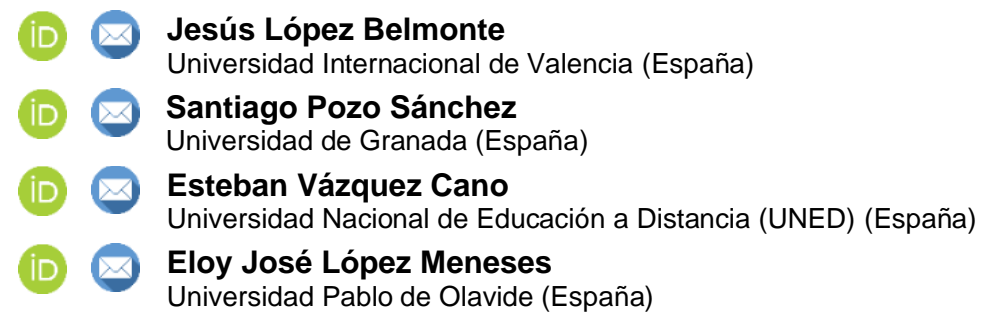

\begin{abstract}
Resumen
Este artículo presenta una investigación en la que analiza la incidencia de la edad en la competencia digital de 611 docentes españoles preuniversitarios. La muestra se ha obtenido a través de la técnica de muestreo por estratos en diferentes centros educativos públicos, concertados y privados españoles en ocho comunidades autónomas. A través de una metodología cuantitativa se han aplicado la prueba Chi-cuadrado de Pearson $(x 2)$ para la comparación entre las distintas variables presentadas y la prueba V de Cramer (VCramer) para determinar la fuerza de asociación entre las mismas. Los resultados muestran que en las tres primeras áreas de la competencia digital percibida: "Información y alfabetización informacional" (ÁREA1); "Comunicación y colaboración" (ÁREA2) y "Creación de contenidos digitales" (ÁREA3), los docentes que tienen una edad no superior a 40 años poseen un nivel más alto de desarrollo competencial. Con respecto al área de "Seguridad en entornos digitales" (ÁREA4), el grupo entre 40 y 49 años es el que mayor competencia demuestra. Asimismo, en relación con la "Resolución de problemas tecnológicos" (ÁREA5) no se identifican diferencias significativas entre los diferentes grupos de edad.
\end{abstract}

\begin{abstract}
This article presents a research that analyzes the incidence of age in the digital competence of 611 pre-university Spanish teachers. The sample has been obtained through the sampling technique by strata in different public, private and Spanish educational centers in eight autonomous Communities. Through a quantitative methodology, the Chi-square Pearson test (X2) was applied to compare the different variables presented and the Cramer $V$ test (VCramer) to determine the strength of association between them. The results show that in the first three areas of perceived digital competence: "Information and information literacy" (AREA1); "Communication and collaboration" (ÁREA2) and "Creation of digital content" (ÁREA3), teachers who are no older than 40 years have a higher level of digital competence. With respect to the area of "Security in digital environments" (AREA4), the group between 40 and 49 years old is the one that shows the greatest competence. Also, in relation to the "Resolution of technological problems" (AREA5) no significant differences are identified between the different age groups.
\end{abstract}

\section{Palabras clave / Keywords}

competencia digital, profesorado preuniversitario, España, edad.

digital competence, pre-university teachers, Spain, age. 


\section{Introducción}

El siglo XXI es el siglo de la información donde se concibe el aprendizaje individual principalmente a través de tecnologías interactivas y participativas que ofrezcan una experiencia estimulante y socialmente positiva, pero que, al tiempo, se conviertan en un constructo que permita al alumnado aprender haciendo, a la vez que comparte sus experiencias de conocimiento con otros que, frecuentemente, se encuentran al otro lado de ese espacio virtual (Cabero, Vázquez-Cano, \& López Meneses, 2018; Tafazoli, Huertas Abril, \& Gómez Parra, 2019). En este sentido, es indudable que la tecnología tiene constante presencia en nuestra vida actual, predominando las redes sociales como contextos de relación o los repositorios de contenidos como espacios de colaboración que evidencian el desarrollo de la creatividad de sus usuarios (De Pablos, 2013, 2015; Sevillano \& Vázquez-Cano, 2015; López-Gil \& Bernal-Bravo, 2019).

Vivimos en una sociedad, que por su dinámica actual, precisa que los sistemas educativos alrededor del mundo replanteen la visión de los futuros profesionales que desean formarse acorde al desarrollo económico global y las exigencias del mercado laboral cada vez más cambiante; haciendo a un lado el modelo de enseñanza tradicional basado en la trasmisión y memorización de conocimientos, en pro de otras metodologías que permitan a los estudiantes adquirir un conjunto de conocimientos, habilidades y actitudes para aplicarlos en un escenario laboral cercano y realista (Fernández, Leiva, \& López Meneses, 2017). A su vez, las funciones socioeducativas de la educación actual deben orientarse a preparar a los futuros ciudadanos para comprender e interpretar la complejidad política, económica y cultural, navegar en la incertidumbre, desarrollar empleos desconocidos hasta ahora, participar en la vida colectiva de un mundo global y local en cambios vertiginosos y permanentes (De Pablos et al., 2017; Pérez-Gómez, 2015).

En última instancia, las competencias en materia relativa a las tecnologías de la información y la comunicación (TIC) en la sociedad actual son imprescindibles para el desarrollo de una ciudadanía activa para la formación de los futuros docentes (García-Vera, García, \& Roig-Vila, 2016; Marín-Díaz \& Reche, 2012; Ríos \& Gómez, 2013). Asimismo, esta nueva sociedad digital demanda nuevos profesionales con una amplia gama de competencias, entre ellas, la denominada competencia digital (Barceló Cerdá \& Ruíz-Corbella, 2015; MarínDíaz et al, 2018).

\section{Las competencias digitales en docentes}

El desarrollo de las tecnologías de la información y la comunicación (TIC), han supuesto dentro de la sociedad una revolución que ha ido marcando el devenir de la vida educativa de los individuos. Esta circunstancia ha puesto de manifiesto la necesidad de proporcionar a los docentes de todos los niveles educativos de una formación que les capacite para la inclusión de las tecnologías en su metodología de aula (Marín-Díaz et al., 2018). En concordancia con Agabo (2015) consideramos que las TIC no son únicamente artefactos que sustituyen las estrategias didácticas en el proceso educativo, sino que pueden ser consideradas como instrumentos que permiten apoyar nuevas formas de enseñar y aprender.

Actualmente, la preocupación por la adquisición de las competencias, que tiene su origen en el mundo laboral, ha trascendido al mundo académico (García-Vera et al., 2016). Este es un paso normal, partiendo de la base de que una función destacada de la universidad es capacitar y formar al individuo para que pueda acceder y desenvolverse en el mundo laboral globalizado y en la sociedad en general (Rodríguez et al., 2010).

Asimismo, la formación del profesorado no debe estar ajena a los cambios que ocurren en la sociedad del siglo XXI. Esta debe estar en sintonía con los mismos y preparar a los futuros docentes para que sean capaces de incluir las tecnologías digitales de forma pertinente, crítica y reflexiva en sus prácticas formativa (Cabrera et al. 2018; Fombona Cadavieco, Vázquez-Cano, \& Del Valle Mejías, 2018). En concordancia con Moreno (2017), es necesario renovar el contenido y los métodos pedagógicos en simbiosis con el docente que debe adquirir competencias para desenvolverse en los entornos tecnológicos. En este sentido, diferentes autores han definido una competencia como un proceso en el cual las personas pueden resolver creativamente problemas, realizar actividades, formular preguntas, buscar información relevante, analizar, comprender y reflexionar al aplicar su conocimiento dando una respuesta a las demandas de un entorno real (Ramos, Chiva, \& Gómez, 2017; Serrano, Biedermann, \& Santolaya, 2016). Se puede decir que nos encontramos frente a un cambio paradigmático respecto al proceso de formación de profesionales (López-Meneses \& Vázquez-Cano, 2013). A su vez, se puede explicitar por competencia digital la capacitación de saber utilizar la tecnología de manera eficaz y supone un compendio de destrezas, habilidades y actitudes ante diferentes áreas y dimensiones de conocimiento (Vázquez-Cano, 2015). En este sentido, la competencia digital se incluye en 
los listados competenciales de las diferentes instituciones oficiales (Comisión Europea, 2006, 2016; INTEF, 2017; OCDE, 2005; UNESCO, 2011).

Por su parte, el Instituto Nacional de Tecnologías Educativas y de Formación del Profesorado (2017), expresa que la: la Competencia digital implica el uso crítico y seguro de las Tecnologías de la Sociedad de la Información para el trabajo, el tiempo libre y la comunicación. Apoyándose en habilidades TIC básicas: uso de ordenadores para recuperar, evaluar, almacenar, producir, presentar e intercambiar información, y para comunicar y participar en redes de colaboración a través de Internet. (INTEF, 2017, p.8). Asimismo, en el marco de la Recomendación del Parlamento Europeo y del Consejo de 18 de diciembre de 2006 sobre las competencias clave para el aprendizaje permanente (2006/962/CE) se indica que: La competencia digital entraña el uso seguro y crítico de las tecnologías de la sociedad de la información (TSI) para el trabajo, el ocio y la comunicación. Se sustenta en las competencias básicas en materia de TIC: el uso de ordenadores para obtener, evaluar, almacenar, producir, presentar e intercambiar información, y comunicarse y participar en redes de colaboración a través de Internet (Parlamento Europeo y Consejo de la Unión Europea, 2006, p. 15). Por último, puede destacarse el modelo más holístico e integrador (conocimientos-habilidades-actitudes) de competencia digital el DigComp (Comisión Europea, 2014, 2016; Ferrari, Neza, \& Punie, 2014).

En un enfoque educativo por competencias, se requiere plantear mecanismos que busquen transformar la enseñanza y el aprendizaje (Barbosa \& Amariles, 2019). La enseñanza a través de las prácticas docentes que permiten ofrecer una enseñanza contextualizada eliminando las brechas entre la universidad y la sociedad (Unigarro, 2017). Es por ello que, bajo este escenario tecno-educativo, debe venir acompañado de una formación y, por consiguiente, una actualización tecnopedagógica de las habilidades y conocimientos en materia digital que el profesorado requiere para desplegar su labor en una era digitalizada (Area, 2010).

En esta línea argumental competencial, el Instituto Nacional de Tecnologías Educativas y de Formación del Profesorado (INTEF, en adelante), con el deseo intencional de regularizar y normalizar las destrezas competenciales profesionales a nivel tecnológico que debe reunir un docente en la educación actual, ha establecido 5 áreas que vinculan la competencia digital. Estas quedan recogidas de la siguiente manera (INTEF, 2017):

1) Información y alfabetización informacional.

2) Comunicación y colaboración.

3) Creación de contenidos digitales.

4) Seguridad.

5) Resolución de problemas.

Durante los últimos años se viene evidenciando la utilidad de las TIC en determinados procesos de enseñanza-aprendizaje, en el aumento de la motivación de los estudiantes y de su curiosidad (Cox \& Marshall, 2007; Livingstone, 2012). En muchos casos, el uso de la tecnología en el aula para el desarrollo de competencias y de procesos de evaluación viene evidenciado que promueve el autoaprendizaje y el desarrollo de la competencia de aprender a aprender (Area \& Ribeiro-Pessoa, 2012; Krumsvik, 2008; Passey et al., 2004). Por este motivo, que el profesorado sea competente en el uso situado de la tecnología en su contexto escolar es un reto y requerimiento de todo sistema educativo (Vázquez-Cano, 2016). Para ello, la formación continua del profesorado es una necesidad, como la de cualquier profesional en cualquier campo especializado. Los nuevos enfoques didácticos basados en el trabajo más colaborativo y competencial del estudiante junto con nuevas formas de evaluar el desempeño académico del alumnado son requisitos del docente del siglo XXI y, en donde la edad, no debe suponer un hándicap para su desarrollo.

A pesar de la incidencia tecnológica, determinados estudios han reflejado que el profesorado no se encuentra preparado ni formado para efectuar sus funciones de manera eficaz (Afanador, 2017; Fernández, Leiva, \& López, 2018). Y de este modo, como apuntan Cabero y Ruiz (2018), se produce una brecha entre los discentes y docentes (los primeros entendidos como nativos y los segundos como inmigrantes digitales).

Por lo tanto, este estudio intenta determinar la dificultad que puede entrañar la edad para desarrollar y consolidar una adecuada competencia digital en los procesos de enseñanza-aprendizaje en cinco áreas prioritarias: (1) Información y alfabetización informacional. (2) Comunicación y colaboración. (3) Creación de contenidos digitales. (4). Seguridad en entornos digitales y (5) Resolución de problemas tecnológicos. El contexto normativo actual y las competencias docentes actuales requieren la figura de un docente que demuestre una adecuada competencia digital que le permite afrontar con garantías una educación de calidad. La literatura publicada hasta el momento no aborda estas variables de forma interrelacionada; por lo que su estudio puede aportar evidencias con hondas implicaciones en la formación del profesorado, en el desarrollo 
de las asignaturas y competencias del alumnado y en la consecución de los elementos transversales del currículo.

\section{Metodología}

Esta investigación tiene su razón de ser en la consecución del objetivo "conocer el estado de la competencia digital en docentes españoles según su intervalo de edad". De este enunciado de índole general han derivado los siguientes objetivos con mayor escala de especificación:

- Determinar el nivel de competencia digital docente en cada una de las 5 áreas que la conforman en base a la edad de los profesionales.

1) Concretar el grado competencial concerniente a la información y alfabetización informacional conforme al intervalo de edad.

2) Averiguar el estado competencial relacionado a la comunicación y colaboración de acuerdo con el rango de edad.

3) Descubrir el índice competencial referido a la creación de contenidos digitales con arreglo al intervalo de edad.

4) Conocer el nivel competencial conexo a la seguridad en entornos digitales según el rango de edad.

5) Indagar en el grado competencial afín a la resolución de problemas tecnológicos a proporción del intervalo de edad.

En base a las orientaciones metodológicas de Hernández, Fernández y Baptista (2014), para lograr el alcance de los objetivos formulados, el presente estudio se ha desarrollado siguiendo un método cuantitativo, por medio de un diseño de investigación no experimental de corte descriptivo y correlacional.

\subsection{Participantes}

La muestra de estudio ha sido compuesta por 611 docentes españoles, de los cuales el $40.26 \%$ son mujeres y el $59.74 \%$ son hombres, pertenecientes a diferentes tipologías de centros educativos (centros públicos $=60.72 \%$; centros concertados $=30.44 \%$; centros privados $=8.84 \%$ ), así como de diversas Comunidades y Ciudades Autónomas de España (Andalucía $=22.26 \%$; Castilla y León=8.84\%; Madrid=27.5\%; Galicia=11.62\%; Aragón=5.73\%; Comunidad Valenciana=6.87\%; Extremadura=3.44\%; Ceuta=13.75\%).

Estos sujetos han sido seleccionados a través de la técnica de muestreo por estratos y -posteriormente- en cadena, o también denominada por bola de nieve (Bisquerra, 2004). Los profesionales que han participado en este estudio se encuentran detallados en la Tabla 1 según su edad ( $\mathrm{Me}_{\mathrm{e}} 39.86$; DT=10.49).

\section{Tabla 1}

Intervalos de edades establecidos

\begin{tabular}{lll}
\hline Intervalo & $\mathbf{n}$ & $\%$ \\
\hline Entre 21 y 29 años & 56 & 9.16 \\
Entre 30 y 39 años & 261 & 42.71 \\
Entre 40 y 49 años & 148 & 24.22 \\
Entre 50 y 59 años & 107 & 17.51 \\
Más de 59 años & 39 & 6.38 \\
Total & 611 & 100 \\
\hline
\end{tabular}

\subsection{Instrumento}

La recogida de datos se ha efectuado a través de un cuestionario "ad hoc" y que se puede consultar en la siguiente dirección: https://forms.gle/SezUQVeNEJYwpUo1A. Su confección parte del análisis de otros instrumentos (Agreda, Hinojo, \& Sola, 2016; López Meneses, Leiva, J.J., \& Vázquez-Cano, 2017; Tourón et al., 2018). El instrumento diseñado se compone de 64 ítems, clasificados en 6 dimensiones: Social (SOC); Área 1 de la CDD (INFOR); Área 2 de la CDD (COMCOL); Área 3 de la CDD (CRECON); Área 4 de la CDD 
(SEG); Área 5 de la CDD (RESOL). El cuestionario sigue un formato de respuesta en escala Likert de 1-4 donde 1 es el valor más negativo y 4 el más positivo- y otras de elección cerrada.

Siguiendo las orientaciones de McMillan y Schumacher (2005), el cuestionario fue sometido a doble validación antes de su aplicación en el trabajo de campo. La primera -a nivel cualitativa- mediante la técnica de juicio de expertos (14 doctores en tecnología educativa) cuyo feedback fue contrastado por estadísticos (Kappa de Fleiss, $\mathrm{K}=.827$; $\mathrm{W}$ de Kendall, $\mathrm{W}=.863$ ) resultando concordante y pertinente para mejorar la herramienta.

La segunda validación fue cuantitativa en una muestra independiente de 328 sujetos, en la que se empleó el test de Kaiser-Meyer-Olkin (KMO=.892) y el test de esfericidad de Bartlett $(p=.0005)$, dando lugar a un nivel de adecuación alto en el análisis de componentes principales (ACP). Los ítems presentaron una asimetría negativa y una curtosis moderada, por lo que se efectuó un contraste de normalidad (Prueba de KolmogorovSmirnov) con corrección Lilliefors, alcanzando valores adecuados. Asimismo, el coeficiente de Mardia reveló una normalidad multivariante. Del mismo modo, la estructura interna se analizó mediante un análisis factorial confirmatorio (AFC) con la técnica de máxima verosimilitud y rotación Varimax, siendo los parámetros estimados estadísticamente significativos y con saturaciones elevadas. Los índices de ajuste utilizados (bondad de Joreskog, normado de Bentler-Bonett, comparativo de Bentler y el error cuadrático medio de aproximación) arrojaron valores adecuados, originando un modelo sustentable.

La confiabilidad del instrumento se determinó por el Alfa de Cronbach, alcanzándose los siguientes valores: INFOR: $\alpha=.817$; COMCOL: $\alpha=.835$; CRECON: $\alpha=.803$; SEG: $\alpha=.844$; RESOL: $\alpha=.826$. Estos valores originan un $\alpha=.825$ en la globalidad del instrumento, considerándose una fiabilidad relevante.

\subsection{Variables de estudio}

Las variables que se han definido en este estudio -junto con su abreviatura- se encuentran recogidas en la Tabla 2, con el propósito de agilizar su lectura e interpretación de los datos.

\section{Tabla 2}

Variables de la investigación

\begin{tabular}{ll}
\hline Variables & Abreviatura \\
\hline Competencia digital docente & CDD \\
Información y alfabetización informacional & ÁREA1 \\
Comunicación y colaboración & ÁREA2 \\
Creación de contenidos digitales & ÁREA3 \\
Seguridad en entornos digitales & ÁREA4 \\
Resolución de problemas tecnológicos & ÁREA5 \\
$21-29$ años de edad & IAGE1 \\
$30-39$ años de edad & IAGE2 \\
$40-49$ años de edad & IAGE3 \\
$50-59$ años de edad & IAGE4 \\
Más de 59 años de edad & IAGE5
\end{tabular}

\subsection{Procedimiento}

El estudio tuvo su origen en el mes febrero de 2018 con la confección del cuestionario y su posterior proceso de validación. Acto seguido, en el mes de marzo de 2018 se produjo la creación del cuestionario en línea y su posterior envío a los distintos centros educativos gracias a la base de datos proporcionada por el Ministerio de Educación y Formación Profesional de España. Para la selección de los centros educativos se llevó a cabo un muestreo aleatorio por estratos.

Una vez escogidos, se produjo un primer acercamiento con los coordinadores TIC, con el propósito de hacer llegar el instrumento a los distintos docentes. A su vez, cada participante debía rellenar en un campo del cuestionario la dirección de correo electrónico de otros 3 profesionales de la educación procurando ser de diferentes centros educativos, con el fin de expandir la difusión del instrumento por el mecanismo de bola de nieve.

Para el proceso de recogida de información se estableció una duración de 8 meses (marzo-octubre de 2018), al coincidir algunas fechas con periodo vacacional lo que supuso un retraso en el proceso. Transcurrido ese 
tiempo, se dio por concluida la fase de recolección y se procedió a la preparación de los datos para iniciar un análisis estadístico en profundidad.

\subsection{Análisis de datos}

Para valorar la competencia digital docente se han tomado estadísticos como la media (M), la desviación típica (DT), el coeficiente de asimetría de Pearson (CAP) y el coeficiente de asimetría de Fisher (CAF). Asimismo, la prueba Chi-cuadrado de Pearson (X2) para la comparación entre las distintas variables presentadas y la prueba $\mathrm{V}$ de Cramer $(\mathrm{V}$ cramer) para determinar la fuerza de asociación entre las mismas. El programa utilizado para el tratamiento estadístico de los datos ha sido el Statistical Package for the Social Sciences (SPSS) v.22, considerando un $p<0.05$ como diferencia estadísticamente significativa.

\section{Resultados}

En lo referente al descriptivo pormenorizado del nivel competencial referenciado por los docentes en material digital (Tabla 3), los resultados más positivos se contabilizan en el grupo de menor edad (IAGE1), produciéndose un decrecimiento progresivo hasta llegar al grupo interválico de edad más avanzada (IAGE 5), el cual presenta los niveles más negativos en competencia digital. Por otra parte, los estadísticos realizados para el estudio de la asimetría (Coeficientes de asimetría de Fisher y Pearson) muestran una distribución totalmente asimétrica negativa para los tres primeros grupos interválicos de edad (IAGE1, IAGE2 e IAGE3) y una distribución asimétrica positiva casi en su totalidad para los grupos de edad más avanzada (IAGE4 e IAGE5). De esta forma, se observa una tendencia inversamente proporcional entre la edad de los docentes y el nivel competencial mostrado.

Tabla 3

Resultados obtenidos en las cinco áreas de la competencia digital

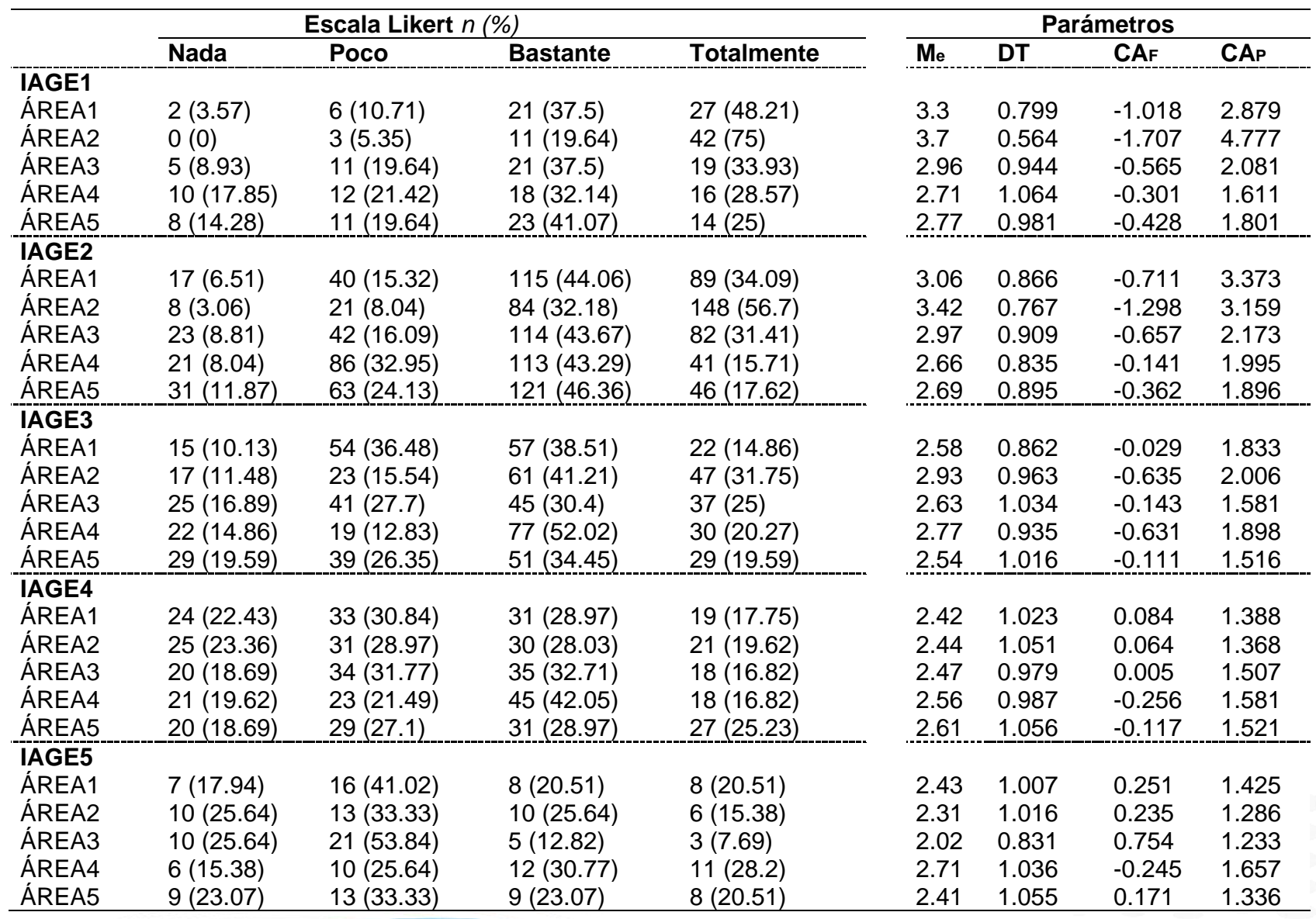


Poniendo en valor los diferentes resultados obtenidos en torno al promedio de tendencia central de cada uno de los grupos de edad analizados (Figura 1) los resultados muestran la existencia de importantes diferencias en el nivel promedio obtenido por los diferentes grupos de edad en las tres primeras áreas de estudio (ÁREA1, ÁREA 2 y ÁREA3), siendo especialmente acuciante la diferencia de las medias en el área relacionada con la comunicación (RangoÁrEA2=1.39). Dichas diferencias se reducen ostensiblemente en los resultados del resto de áreas (ÁREA4 y ÁREA5), evidenciándose puntuaciones similares en el promedio de los diferentes grupos de edad (RangoÁREA4= 0.21; RangoÁREA5=0.36). Las puntuaciones medias más altas han sido obtenidas por los grupos de menor edad (IAGE1 e IAGE2), resultados que contrastan notablemente con los obtenidos por el grupo de edad más avanzada (IAGE5).

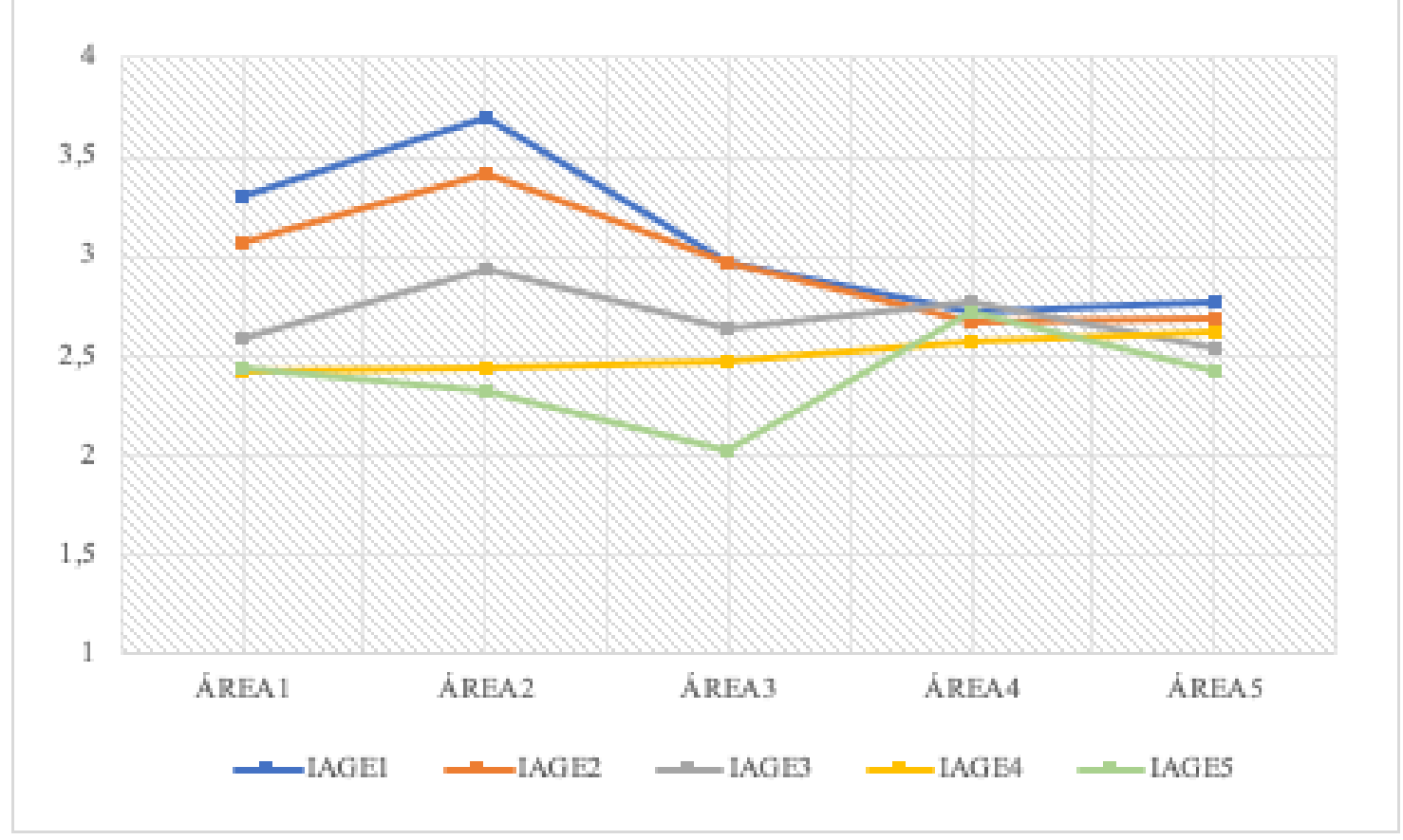

Figura 1. Comparación de medias según el nivel competencial y el intervalo de edad.

Los resultados concernientes al área de información y alfabetización informacional (Tabla 4) muestran que los dos grupos de edad más reducida (IAGE1 e IAGE2) presentan niveles superiores en la citada área. De esta forma, los datos paramétricos evidencian la existencia de una asociación significativa $(p$-valor $<0.05)$ entre la edad de los docentes y su nivel competencial en lo que dicha área se refiere. Los estadísticos de interpretación del tamaño del efecto determinan que nos encontramos ante un alto nivel de asociación (V> 0.6).

Tabla 4

Asociación entre el intervalo de edad y el nivel competencial del ÁREA1

\begin{tabular}{|c|c|c|c|c|c|c|c|c|c|}
\hline \multirow[b]{2}{*}{ Likert } & \multicolumn{4}{|c|}{ Intervalo de edad $n(\%)$} & \multirow[b]{2}{*}{ IAGE5 } & \multicolumn{4}{|c|}{ Parámetros } \\
\hline & IAGE1 & IAGE2 & IAGE3 & IAGE4 & & $x^{2}(g l)$ & $p$-valor & Cont. & Vcramer \\
\hline ÁREA1 & & & & & & $85.25(12)$ & $<0.001$ & 0.351 & 0.647 \\
\hline Nada & $2(0.32)$ & $17(2.78)$ & $15(2.45)$ & 24 (3.92) & 7 (1.14) & & & & \\
\hline Poco & $6(28.8)$ & $40(6.54)$ & $54(8.83)$ & $33(5.4)$ & $16(2.6)$ & & & & \\
\hline Bastante & $21(3.43)$ & $115(18.8)$ & $57(9.32)$ & $31(5.07)$ & $8(1.31)$ & & & & \\
\hline Totalmente & $27(4.41)$ & $89(14.56)$ & $22(3.6)$ & $19(3.11)$ & $8(1.31)$ & & & & \\
\hline
\end{tabular}

En el área de comunicación y colaboración (Tabla 5) nuevamente obtienen los índices más altos de competencia digital los primeros grupos interválicos de edad (IAGE e IAGE2), seguidos del tercer grupo (IAGE3) para el que se han obtenido niveles competenciales intermedios. La valoración paramétrica de la 
asociación muestra la existencia de significancia estadística con un alto nivel de asociación en lo que respecta al tamaño del efecto.

\section{Tabla 5}

Asociación entre el intervalo de edad y el nivel competencial del ÁREA2

\begin{tabular}{|c|c|c|c|c|c|c|c|c|c|}
\hline \multirow[b]{2}{*}{ Likert } & \multicolumn{4}{|c|}{ Intervalo de edad $n(\%)$} & \multirow[b]{2}{*}{ IAGE5 } & \multicolumn{4}{|c|}{ Parámetros } \\
\hline & IAGE1 & IAGE2 & IAGE3 & IAGE4 & & $x^{2}(g l)$ & $p$-valor & Cont. & Vcramer \\
\hline ÁREA2 & & & & & & $139.7(12)$ & $<0.001$ & 0.431 & 0.828 \\
\hline Nada & $0(0)$ & $8(1.31)$ & $17(2.78)$ & $25(4.09)$ & $10(1.63)$ & & & & \\
\hline Poco & $3(0.49)$ & $21(3.43)$ & $23(3.76)$ & $31(5.07)$ & $13(2.12)$ & & & & \\
\hline Bastante & $11(1.8)$ & $84(13.7)$ & $61(9.98)$ & $30(4.91)$ & $10(1.63)$ & & & & \\
\hline Totalmente & $42(6.87)$ & $148(24.2)$ & $47(7.69)$ & $21(3.43)$ & $6(0.98)$ & & & & \\
\hline
\end{tabular}

Existe una relación estadísticamente significativa entre la edad de los docentes y el nivel competencial mostrado en la creación de contenidos digitales (Tabla 6), obteniéndose una fuerza de asociación media entre las variables $(0.3<\mathrm{V}<0.6)$. Los dos primeros grupos de edad (IAGE1 e IAGE2) muestran los niveles más altos de competencia en dicha área. En este caso, es el segundo grupo interválico (IAGE2) el que alcanza el promedio más alto, aunque con una diferencia ínfima con respecto al primer grupo de edad (IAGE).

\section{Tabla 6}

Asociación entre el intervalo de edad y el nivel competencial del ÁREA3

\begin{tabular}{|c|c|c|c|c|c|c|c|c|c|}
\hline \multirow[b]{2}{*}{ Likert } & \multicolumn{4}{|c|}{ Intervalo de edad $n(\%)$} & \multirow[b]{2}{*}{ IAGE5 } & \multicolumn{4}{|c|}{ Parámetros } \\
\hline & IAGE1 & IAGE2 & IAGE3 & IAGE4 & & $x^{2}(g l)$ & $p$-valor & Cont. & Vcramer \\
\hline ÁREA3 & & & & & & $62.09(12)$ & $<0.001$ & 0.304 & 0.552 \\
\hline Nada & $5(0.81)$ & 23 (3.76) & $25(4.09)$ & 20 (3.27) & $10(1.63)$ & & & & \\
\hline Poco & $11(1.8)$ & $42(6.87)$ & $41(6.71)$ & $34(5.56)$ & $21(3.43)$ & & & & \\
\hline Bastante & $21(3.43)$ & $114(18.7)$ & $45(7.36)$ & $35(5.72)$ & $5(0.81)$ & & & & \\
\hline Totalmente & $19(3.11)$ & $82(13.42)$ & $37(6.05)$ & $18(2.94)$ & $3(0.49)$ & & & & \\
\hline
\end{tabular}

Los resultados obtenidos en el área de seguridad digital (Tabla 7) muestran la existencia de valores estadísticamente significativos, aunque con una fuerza de asociación media. Si bien el nivel competencial evidenciado por los diferentes grupos de edad muestra progresiones similares, el grupo interválico intermedio (IAGE3) ha mostrado niveles ligeramente superiores que el resto.

\section{Tabla 7}

Asociación entre el intervalo de edad y el nivel competencial del ÁREA4

\begin{tabular}{|c|c|c|c|c|c|c|c|c|c|}
\hline \multirow[b]{2}{*}{ Likert } & \multicolumn{4}{|c|}{ Intervalo de edad $n(\%)$} & \multirow[b]{2}{*}{ IAGE5 } & \multicolumn{4}{|c|}{ Parámetros } \\
\hline & IAGE1 & IAGE2 & IAGE3 & IAGE4 & & $x^{2}(g l)$ & $p$-valor & Cont. & V Cramer \\
\hline ÁREA4 & & & & & & $38.45(12)$ & $<0.001$ & 0.243 & 0.434 \\
\hline Nada & $10(1.63)$ & $21(3.43)$ & $22(3.6)$ & $21(3.43)$ & $6(0.98)$ & & & & \\
\hline Poco & $12(1.96)$ & $86(14.08)$ & $19(3.11)$ & $23(3.76)$ & $10(1.63)$ & & & & \\
\hline Bastante & $18(2.94)$ & $113(18.5)$ & $77(12.6)$ & $45(7.36)$ & $12(1.96)$ & & & & \\
\hline Totalmente & $16(2.61)$ & $41(6.71)$ & $30(4.91)$ & $18(2.94)$ & $11(1.8)$ & & & & \\
\hline
\end{tabular}

Por último, no se contempla una asociación estadísticamente significativa entre la edad y el nivel competencial mostrado en el área de resolución de problemas (Tabla 8), ya que los resultados de los diferentes grupos de edad muestran niveles competenciales similares atendiendo al grado de libertad de los valores estudiados, constatado estadísticamente por los bajos niveles obtenidos en la corrección aplicada al estadístico Chicuadrado ( $p$-valor $>0.05)$. 
Tabla 8

Asociación entre el intervalo de edad y el nivel competencial del ÁREA5

\begin{tabular}{|c|c|c|c|c|c|c|c|c|c|}
\hline \multirow[b]{2}{*}{ Likert } & \multicolumn{4}{|c|}{ Intervalo de edad $n(\%)$} & \multirow[b]{2}{*}{ IAGE5 } & \multicolumn{4}{|c|}{ Parámetros } \\
\hline & IAGE1 & IAGE2 & IAGE3 & IAGE4 & & $x^{2}(g l)$ & $p$-valor & Cont. & Vcramer \\
\hline ÁREA5 & & & & & & $20.5(12)$ & 0.057 & 0.181 & 0.318 \\
\hline Nada & $8(1.31)$ & $31(5.07)$ & $29(4.74)$ & $20(3.27)$ & $9(1.47)$ & & & & \\
\hline Poco & $11(1.8)$ & $63(10.3)$ & $39(6.38)$ & $29(4.74)$ & $13(2.12)$ & & & & \\
\hline Bastante & $23(3.76)$ & $121(19.8)$ & $51(8.34)$ & $31(5.07)$ & $9(1.47)$ & & & & \\
\hline Totalmente & $14(2.29)$ & $46(7.52)$ & $29(4.74)$ & $27(4.41)$ & $8(1.31)$ & & & & \\
\hline
\end{tabular}

\section{Discusión}

Los resultados muestran que en las tres primeras áreas de la competencia digital percibida: "Información y alfabetización informacional" (ÁREA1); "Comunicación y colaboración" (ÁREA2) y "Creación de contenidos digitales" (ÁREA3). Los docentes que tienen una edad no superior a 40 años muestran un nivel más alto de desarrollo competencial. Con respecto al área de "Seguridad en entornos digitales" (ÁREA4), el grupo entre 40 y 49 años es el que mayor competencia demuestra. Asimismo, la en relación con la "Resolución de problemas tecnológicos" (ÁREA5) no se identifican diferencias significativas entre los diferentes grupos de edad.

Estos resultados en parte han sido confirmados por estudios previos. Desde una perspectiva más general, diferentes estudios vienen evidenciando que la competencia digital del profesorado es menor de la esperada (Guillén-Gámez, Mayorga-Fernández, \& Álvarez-García, 2018, 2019; Pinto-Llorente et al., 2017; Sadaf, Newby, \& Ertmer, 2016; Siddiq, Scherer, \& Tondeur, 2016). La edad es una de las variables que ha sido objeto de estudio en la determinación de la competencia digital de los docentes (Cabero \& Barroso, 2016; Gudmundsdottir, \& Hatlevick, 2018; John, 2015; Scherer, Siddiq, \& Teo, 2015), aunque los resultados son contradictorios. Por ejemplo, Cabero y Barroso (2016) coinciden con los resultados de este estudio con referencia la mayor competencia digital de los docentes más jóvenes. Asimismo, en un estudio realizado en el contexto noruego se evidenció que los docentes en los primeros años de ejercicio y más jóvenes eran los que mostraban una mayor disposición al empleo pedagógico y didácticas de la tecnología en las aulas (Gudmundsdottir, \& Hatlevik, 2018). Por el contrario, otros investigadores han identificado la franja de edad entre 30-50 como la más dinámica a hora de afrontar una pedagogía mediada pro la tecnología (John, 2015; Siddiq et al., 2016). Si bien, otros estudios muestran que más que la edad como variable determinante influye la motivación (Blackwell, et al., 2013; Tømte, Enochsson, Buskqvist, \& Kårstein, 2015) y también la formación (Vázquez-Cano, 2015).

En este sentido, uno de los aspectos clave que se debería reconsiderar es el binomio dotación tecnológica y formación del profesorado. En muchas ocasiones, la dotación en recursos tecnológicos no ha sido programada de forma paralela a procesos intensos de formación del profesorado y, en más de una ocasión, pizarras digitales, ordenadores portátiles o grandes infraestructuras como la emprendida por la Junta de Extremadura a comienzo del siglo XXI no cuajaron o se desaprovecharon por una deficiente formación del profesorado (Vázquez-Cano, 2017).

Existe también una variable que en determinados estudios ha resultado ser determinante para la falta de adopción de metodologías con base en tecnologías emergentes, el tiempo de experiencia docente. Así, estudios como los de Romero (2004) muestran que a mayor experiencia docente y no necesariamente mayor edad, se apuesta por métodos de enseñanza-aprendizaje más tradicionales o con menor peso tecnológico. Muchas veces las modas tecnológicas tampoco vienen derivadas de procesos de evaluación rigurosos que permitan evidenciar que su uso mejora métodos más tradicionales de enseñanza-aprendizaje.

Hay que tener casi el 60\% de los docentes españoles de secundaria se encuentran en la franja 30-49 y más de un tercio del profesorado de Educación Primaria y Educación Secundaria en España tiene 50 o más años, mientras que los porcentajes de menores de 30 años no alcanzan el 15\% en ningún caso (OCDE, 2019). Los resultados de este estudio impelen a los responsables políticos a la creación de itinerarios formativos que permitan un mayor reciclaje profesional. No es suficiente con una adecuada formación de acceso a la docencia, el mundo de las tecnologías es tan cambiante que se precisa una formación continua, actualizada y vinculada a itinerarios formativos como la seguridad en red, la creación de contenidos y los procesos de colaboración en red. Por ejemplo, la Ley Orgánica Ley Orgánica 3/2018, de 5 de diciembre, de Protección de Datos Personales y garantía de los derechos digitales establece en su artículo 83 el "Derecho a la educación 
digital". Para ello, las administraciones educativas deberán incluir en el diseño del bloque de asignaturas de libre configuración la competencia digital a la que se refiere el apartado anterior, así como los elementos relacionados con las situaciones de riesgo derivadas de la inadecuada utilización de las TIC, con especial atención a las situaciones de violencia en la red. Esto genera una obligación del profesorado a la hora de impartir docencia que debe contemplar una adecuada formación y, derivado de los resultados obtenidos, deberá tener una mayor intensidad en el profesorado entre 30 y 50 años.

Asimismo, es necesario evaluar y analizar el desempeño del profesorado en relación con el empleo de las TIC para el fomento de los elementos transversales del currículo y cómo se afronta de manera colaborativa y coordinada entre las diferentes asignaturas, teniendo en cuenta que la propia LOMCE (2013) estable que "Sin perjuicio de su tratamiento específico en algunas de las materias de este curso, la comprensión lectora, la expresión oral y escrita, la comunicación audiovisual, las Tecnologías de la Información y la Comunicación, el emprendimiento y la educación cívica y constitucional se trabajarán en todas las materias". En este sentido, los servicios de inspección de educación tienen un cometido esencial a la hora de arbitrar actuaciones prioritarias que permitan observar el grado de cumplimiento del currículum y el de las competencias digitales del estudiante. Derivadas de estas actuaciones, se pueden identificar las lagunas principales y diseños planes formativos adecuados a la situación actual tan condicionada por el uso de las tecnologías no siempre desde una perspectiva académica y profesional.

Por lo tanto, no es la edad sino la falta de formación continua del profesorado lo que puede suponer hoy más que nunca un hándicap para el desarrollo efectivo de la competencia digital del estudiante preuniversitario en las diferentes dimensiones e indicadores que la complementan; así, como en el desarrollo curricular de las materias.

\section{Conclusión}

Las diferencias de edad entre el profesorado es una variable que suscita en muchas ocasiones diferencias significativas en ámbitos metodológicos y, especialmente, en relación a cambios en los sistemas de enseñanza-aprendizaje. El conocimiento y aplicación de las tecnologías a los procesos educativos es uno de esos ámbitos donde la edad más se ha documentado como una variable diferenciadora en las actitudes y acciones de los docentes en las aulas (Cabero \& Barroso, 2016; Gudmundsdottir, \& Hatlevick, 2018; John, 2015; Scherer, Siddiq, \& Teo, 2015). Como hemos evidenciado en este estudio, cuando el profesorado sobrepasa los 40 años, se siente menos competente y menos motivado para emplear tecnologías en los ámbitos de la información, alfabetización informacional y creación de contenido educativo digital. Estas áreas son cruciales para un adecuado desarrollo de las competencias clave en las diferentes etapas educativas preuniversitarias, especialmente para el desarrollo de la competencia digital y los elementos transversales del currículo (LOMCE, 2013).

Por ese motivo, la formación del profesorado a partir de determinadas edades debería potenciarse de forma más acusada a través de programas específicos de actualización en metodologías innovadoras y del uso de las tecnologías de forma aplicada y educativa fuera y dentro de las aulas. El sistema de formación preuniversitaria tiene un reto que superar y es la voluntariedad en el proceso formativo. Los docentes no tienen la obligación de formarse y se confía en su buena disposición y voluntariedad para ir adquiriendo nuevas competencias y recursos a la hora de afrontar los procesos de enseñanza aprendizaje. En este sentido, se deberían desarrollar itinerarios formativos flexibles presenciales y en línea, pero de obligado cumplimiento que garanticen la adecuada formación del docente para garantizar la ansiada calidad del sistema educativo.

En este sentido, tanto el contexto normativo recientemente publicado (Ley 3/2018), como el diseño del currículo en competencias requiere del profesorado un reciclaje continuo que permita incorporar la tecnología no solo al desarrollo de contenidos específicos de las materias, sino al desarrollo de elementos transversales, al trabajo en equipo y a la administración y organización digital de los centros educativos del siglo XXI. Para ello, la edad no es el problema, sino la falta de formación vinculada a la actividad profesional organizada en itinerarios formativos y exigida como requisito en el acceso y para la consolidación de complementos docentes como los sexenios. Además de garantizar el derecho de los empleados públicos a la formación continua y a la actualización permanente de sus conocimientos y capacidades profesionales, preferentemente en horario laboral (RD 5/2015. Art. 14 "g"). 


\section{Referencias}

Afanador, H. A. (2017). Estado actual de las competencias TIC de docentes. Puente, 9(2), 23-32.

Agabo, I. S. (2015). Factors influencing the use of Information and Communication Technology (ICT). Teaching and Learning Computer Studies in Ohaukwu Local Governament Area of Ebonyi State-Nigeria. Journal of Education and Practice, 6(7), 71-86.

Agreda, M., Hinojo, M. A., \& Sola, J. M. (2016). Diseño y validación de un instrumento para evaluar la competencia digital de los docentes en la Educación Superior española. Pixel-Bit: Revista de Medios y Educación, 49, 39-56. Area, M. (2010). ¿Por qué formar en competencias informacionales y digitales en la educación superior? RUSC, Revista de Universidad y Sociedad del Conocimiento, 7(2), 2-5.

Area, M., \& Ribeiro-Pessoa, M. T. (2012). From Solid to Liquid: New Literacies to the Cultural Changes of Web 2.0. Comunicar, 19, 13-20.

Barbosa, S. H., \& Amariles, M. L. (2019). Learning Styles and the Use of ICT in University Students within a Competency-Based Training Model. Journal of new approaches in educational research (NAER), 8(1), 1-6. https://doi.org/10.7821/naer.2019.1.296

Barceló Cerdá, M. L., \& Ruíz-Corbella, M. (2015). Las competencias profesionales del maestro de primaria desde la perspectiva del tutor del centro de prácticas. Revista Fuentes, 17(1), 17-39.

http://dx.doi.org/10.12795/revistafuentes.2015.i17.01.

Bisquerra, R. (2004). Metodología de la investigación educativa. La Muralla.

Blackwell, C. K., Lauricella, A. R., Wartella, E., Robb, M., \& Schomburg, R. (2013). Adoption and use of technology in early education: The interplay of extrinsic barriers and teacher attitudes. Computers \& Education, 69, 310-319.

https://doi.org/10.1016/j.compedu.2013.07.024

Cabero, J., \& Barroso, J. (2016). ICT teacher training: A view of the TPACK model/Formación del profesorado en TIC: Una visión del modelo TPACK. Cultura y educación, 28(3), 633-663.

https://doi.org/10.1080/ 11356405.2016.1203526

Cabero, J., \& Ruiz, J. (2018). Las Tecnologías de la Información y Comunicación para la inclusión: reformulando la brecha digital. International Journal of Educational Research and Innovation. IJERI, 9, 16-30.

Cabero, J., Vázquez-Cano, E., \& López Meneses, E. (2018). Uso de la realidad aumentada como recurso didáctico en la enseñanza universitaria. Formación Universitaria, 11(1) 25-34. http://dx.doi.org/10.4067/S0718-50062018000100025

Cabrera, C., Cabrera, A., Carámbula, S., Pérez, A., \& Pérez, M. (2018). Tecnologías digitales: análisis de planes de profesorado de Uruguay. Cuadernos de Investigación Educativa, 9, 13-32.

https://doi.org/10.18861/cied.2018.9.2.2858

Comisión Europea (2006). Competencias clave para el aprendizaje permanente. Recomendación 2006/962/CE del Parlamento Europeo y del Consejo, de 18 de diciembre de 2006, sobre las competencias clave para el aprendizaje permanente [Diario Oficial L 394 de 30.12.2006]

Comisión Europea (2016). DigCompOrg. Digitally Competent Educational Organisations. Recuperado de: https://ec.europa.eu/jrc/en/digcomporg

Cox, M. J., \& Marshall, G. (2007). Effects of ICT: Do we know what we should know? Education and Information Technologies, 12, 59-70.

De Pablos, J. (2013). La formación e investigación en el campo de la tecnología educativa. Revista Fuentes, 13, 9-16. De Pablos, J. (2015). Los centros educativos ante el desafío de las tecnologías digitales. Educatio Siglo XXI, 33(3), 259-262.

De Pablos-Pons, J., Colás-Bravo, P., Conde-Jiménez, J., \& Reyes-de Cózar, S. (2017). La competencia digital de los estudiantes de educación no universitaria: variables predictivas. Bordón. Revista de Pedagogía, 69(1), 169-185. Fernández, E., Leiva, J. J., \& López-Meneses, E. (2017). Formación en competencias digitales en la universidad. Percepciones del alumnado. Campus Virtuales, 6(2), 79-89.

Fernández, E., Leiva, J. J., \& López-Meneses, E. (2018). Competencias digitales en docentes de Educación Superior. Revista Digital de Investigación en Docencia Universitaria, 12(1), 213-231.

Ferrari, A., Neza, B., \& Punie, Y. (2014). DIGCOMP: A Framework for Developing and Understanding Digital Competence in Europe. eLearning Papers, 38, 3-17.

Fombona Cadavieco, J., Vázquez-Cano, E., \& Del Valle Mejías (2018). Análisis de la geolocalización y realidad aumentada en dispositivos móviles, propuestas socio-educativas relacionadas con el entorno y las salidas de campo. Profesorado. Revista de Currículum y formación del profesorado, 22(4), 197-222.

https://doi.org/10.30827/profesorado.v22i4.8413

García-Vera, V., García, P., \& Roig-Vila, R. (2016). El concepto de competencia en el contexto del Espacio Europeo de Educación Superior. En Cobos, D., Gómez-Galán, J. G., \& López-Meneses, E. (Coords.), La Educación Superior en el Siglo XXI: Una reflexión desde y para el profesorado (pp. 68-81). UMET Press.

Gudmundsdottir, G. B., \& Hatlevik, O. E. (2018). Newly qualified teachers' professional digital competence: Implications for teacher education. European Journal of Teacher Education, 41(2), 214-231. https://doi.org/10.1080/ 02619768.2017.1416085

Guillén-Gámez, F. D., Lugones, A., \& Mayorga-Fernández, M.a J. (2019). ICT use by pre-service foreign languages teachers according to gender, age and motivation. Cogent Education, 6, 1-17. 
https://doi.org/10.1080/2331186X.2019.1574693

Guillén-Gámez, F. D., Mayorga-Fernández, M. J., \& Álvarez-García, F. J. (2018). A study on the actual use of digital competence in the practicum of education degree. Technology, Knowledge and Learning, 11, 1-16.

https://doi.org/10.1007/s10758-018-9390-z

Hernández, R., Fernández, C., \& Baptista, M. P. (2014). Metodología de la investigación. McGraw Hill.

INTEF (2017). Marco de Competencia Digital. Ministerio de Educación, Ciencia y Deportes.

INTEF, Instituto Nacional de Tecnologías Educativas y de Formación del Profesorado (2017). Organizaciones

Educativas Digitalmente Competentes. Recuperado de http://educalab.es/intef/digcomp/digcompor

John, S. P. (2015). The integration of information technology in higher education: A study of faculty's attitude towards IT adoption in the teaching process. Contaduría y Administración, 60, 230-252.

https://doi.org/10.1016/j.cya.2015.08.004

Krumsvik, R. J. (2008). Situated learning and teachers' digital competence. Education and Information Technologies, 13, 279-290.

Ley Orgánica 8/2013, de 9 de diciembre, para la mejora de la calidad educativa. Boletín Oficial del Estado. Madrid, 10 de diciembre de 2013, núm. 295.

Livingstone, S. (2012). Critical reflections on the benefits of ICT in education. Oxford review of Education, 38, 9-24. López Meneses, E., Leiva, J.J., \& Vázquez-Cano, E. (2017). The impact of personal interactions on the stress of school administrators: The validation and application of an assessment tool. Intangible Capital, 13(3), 499-522. http://dx.doi.org/10.3926/ic.993

López-Gil, M. \& Bernal-Bravo, C. (2019). El perfil del profesorado en la Sociedad Red: reflexiones sobre las competencias digitales de los y las estudiantes en Educación de la Universidad de Cádiz. International Journal of Educational Research and Innovation. IJERI, 11, 83-100.

López-Meneses, E., \& Vázquez-Cano, E. (2013). WEB 2.0 Tools for social Educator training in Higher Education. International Journal of Research in Social Sciences, 3(2), 1-13.

Marín-Díaz, V., \& Reche, E. (2012). Universidad 2.0: actitudes y aptitudes ante las TIC del alumnado de nuevo ingreso de la escuela universitaria de magisterio de la UCO. Píxel-Bit. Revista de Medios y Educación, 40, $197-211$.

Marín-Díaz, V., Burgos-Mellado, S., \& López-Pérez, M. (2018). Formación de docentes para la inclusión digital desde el plan escuela 2.0: estudio de un caso. International Journal of Educational Research and Innovation. IJERI, 10, 274298.

McMillan, J. H., \& Schumacher, S. (2005). Investigación educativa. Madrid: Pearson.

Moreno, E. (2017). Constructos teóricos sobre las competencias tecnológicas del docente universitario para los entornos virtuales de enseñanza y aprendizaje. Encuentros 1(1), 67-85.

OCDE (2005). The definition and selection of key competences. Executive Summary. Recuperado de

http://www.oecd.org/pisa/35070367.pdf

OCDE (2019). Panorama de la Educación: Indicadores de la OCDE. Recuperado de

https://www.educacionyfp.gob.es/dam/jcr:b8f3deec-3fda-4622-befb-

386a4681b299/panorama\%20de\%20la\%20educaci\%C3\%B3n\%202019.pdf

Parlamento Europeo y Consejo de la Unión Europea (2006). Recomendación del Parlamento Europeo y del Consejo de 18 de diciembre de 2006 sobre las competencias clave para el aprendizaje permanente (2006/962/CE). Diario Oficial de la Unión Europea, L 394/10, 30.12.2006. Recuperado de http://bit.ly/2vcTYS9

Passey, D., Rogers, C., Machell, J., McHugh, G., \& Allaway, D. (2004). The Motivational Effect of ICT on Pupils. Lancaster, UK: Department of Educational Research.

Pérez-Gómez, A. (2015). Siete tesis a debate. Cuadernos de Pedagogía, 462, 16-20.

Pinto-Llorente, A. M., Sánchez-Gómez, M. C., García-Peñalvo, F. J., \& Casillas-Martín, S. (2017). Students' perceptions and attitudes towards asynchronous technological tools in blended-learning training to improve grammatical competence in English as a second language. Computers in Human Behavior, 72, 632-643. https://doi.org/10.1016/j.chb.2016.05.071

Ramos, G., Chiva, I., \& Gómez, M. a. B. (2017). Las competencias básicas en la nueva generación de estudiantes universitarios: Una experiencia de Innovación. Revista de Docencia Universitaria, 15(1), 37-55. https://doi.org/10.4995/redu.2017.5909

Ríos, J. M., \& Gómez, E. R. (2013). Relación entre competencias básicas de los estudiantes y competencias del profesorado. Revista Fuentes, 14, 209-230.

Rodríguez, G., Cubero, J., Quesada, V., Ibarra, M. S., Gallego, B., Gómez, M. A. Cabeza, D. (2010). MODES: Desarrollo de competencias interpersonales en Educación Superior a través de juegos de simulación. Comunicación presentada en el VII Simposio Pluridisciplinar sobre Diseño y Evaluación de Contenidos Educativos Reutilizables (SPDECE 2010), Universidad Tecnológica Metropolitana, Mérida, Yucatán, México y Universidad de Cádiz, Andalucía, España.

Sadaf, A., Newby, T. J., \& Ertmer, P. A. (2016). An investigation of the factors that influence preservice teachers' intentions and integration of Web 2.0 tools. Educational Technology Research and Development, 64(1), 37-64. https://doi.org/10.1007/s11423-015-9410-9

Scherer, R., Siddiq, F., \& Teo, T. (2015). Becoming more specific: Measuring and modeling teachers' perceived usefulness of ICT in the context of teaching and learning. Computers \& Education, 88, 202-214. 
https://doi.org/10.1016/j.compedu.2015.05.005

Serrano, T. A., Biedermann, A. M., \& Santolaya, S. J. (2016). Perfil, objetivos, competencias y expectativas de futuro profesional de los estudiantes del Grado en Ingeniería en Diseño Industrial y Desarrollo de Producto de la Universidad de Zaragoza. Revista de Docencia Universitaria, 14(1), 69-96.

https://doi.org/10.4995/ redu.2016.5908

Sevillano, M.-áL., \& Vázquez-Cano, E. (2015). The impact of digital mobile devices in Higher Education. Educational Technology \& Society, 18(1), 106-118.

Siddiq, F., Scherer, R., \& Tondeur, J. (2016). Teachers' emphasis on developing students' digital information and communication skills (TEDDICS): A new construct in 21st century education. Computers \& Education, 92, 1-14. https://doi.org/10.1016/j.compedu.2015.10.006

Tafazoli, D., Huertas Abril, C., \& Gómez Parra, Maㅡ E. (2019). Technology-Based Review on Computer-Assisted Language Learning: A Chronological Perspective. Píxel-Bit. Revista de Medios y Educación, 0(54), 29-44.

http://dx.doi.org/10.12795/pixelbit.2019.i54.02

Tømte, C., Enochsson, A. B., Buskqvist, U., \& Kårstein, A. (2015). Educating online student teachers to master professional digital competence: The TPACK-framework goes online. Computers \& Education, 84, 26-35. https://doi.org/10.1016/j.compedu.2015.01.005

Tourón, J., Martín, D., Navarro, E., Pradas, S., \& Íñigo, V. (2018). Validación de constructo de un instrumento para medir la competencia digital docente de los profesores (CDD). Revista española de pedagogía, 269, 25-54. Unigarro, M. (2017). Un modelo educativo crítico con enfoque de competencias. Colombia: Ediciones Universidad Cooperativa de Colombia.

Vázquez-Cano, E. (2015). El Reto de la formación docente para el uso de dispositivos digitales móviles en la Educación Superior. Perspectiva Educacional, 54(1), 149-162.

Vázquez-Cano, E. (2016). Dificultades del profesorado para planificar, coordinar y evaluar competencias clave. Un análisis desde la Inspección de Educación. Revista Complutense de Educación, 27(3), 1061-1083.

http://doi.org/10.5209/rev_RCED.2016.v27.n3.4740

Vázquez-Cano, E. (2017). Analysis of Difficulties of Spanish Teachers to Improve Students' Digital Reading Competence. A Case Study within the PISA Framework. Pedagogika, 125(1), 175-194.

http://dx.doi.org/10.15823/p.2017.13 